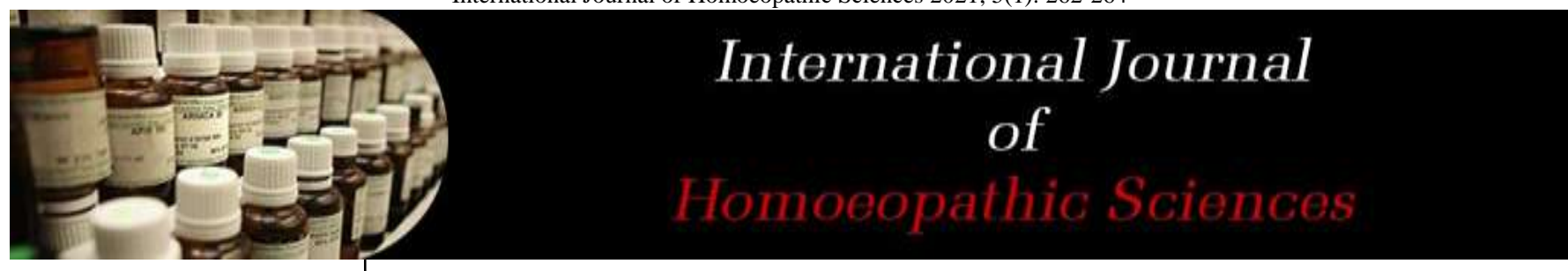

E-ISSN: $2616-4493$ P-ISSN: 2616-4485 www.homoeopathicjournal.com IJHS 2021; 5(1): 262-264 Received: 28-11-2020 Accepted: 09-01-2021

Dr. Gurtej Kaur BHMS, MD (Homeopathy) Scholar, Sri Guru Nanak Dev Homoeopathic Medical College and Hospital, Ludhiana, Punjab, India
Corresponding Author: Dr. Gurtej Kaur BHMS, MD (Homeopathy) Scholar, Sri Guru Nanak Dev Homoeopathic Medical College and Hospital, Ludhiana, Punjab, India

\section{Urinary tract infection and homoeopathy}

\section{Dr. Gurtej Kaur}

DOI: https://doi.org/10.33545/26164485.2021.v5.i1e.322

\section{Abstract}

Urinary tract infection is the most common disorder of the renal tract. Although serious morbidities from UTI are very low but if left untreated that can lead to serious complications. Bacteraemia from UTI can be a life-threatening medical emergency.

Homoeopathy is a holistic medical practice that treats the patient as a whole. Homoeopathic medicines are a useful alternative to conventional medication offering earlier symptom resolution, cost effectiveness and fewer adverse Urinary tract infection and their complications are most frequent cause of antibiotic prescribing. Antibiotic resistance is a global public health problem. The prevalence of resistant bacteria is rising and organisms resistant to almost all antibiotics have been identified. In the light of antimicrobial resistance, homoeopathy offers alternative strategies in combating the development of antibiotic resistance. Homoeopathic medicines show remarkable results in treatment of urinary tract infection, to avoid its recurrence and reduce the chances of complications in a safe and gentle way.

Keywords: Homoeopathy, urinary tract infection, therapeutics

\section{Introduction}

Urinary tract infection' (UTI) refers to bacterial, viral, or fungal infection of the kidneys, renal pelvis, ureters, or bladder. The term UTI constitutes various clinical entities: cystitis, urethritis, pyelonephritis, prostatitis and asymptomatic bacteriuria.

Uncomplicated UTI refers to acute disease occurring in an anatomically and functionally normal urinary tract. Complicated UTI refers to infection of any part of the urinary tract with functional or structural urinary abnormalities. It refers to all infections occurring in patients either with impaired host defence (e.g. diabetes), with abnormal urinary tract function (e.g. pregnancy), or abnormal urinary tract anatomy (e.g. urinary tract obstruction).

\section{Epidemiology}

UTI occurs commonly in females than in males, although obstruction from prostatic hypertrophy and the rising prevalence of impaired bladder emptying leads to an increased incidence of infection causes men $>50$ years old to have an incidence of UTI comparable to that among women of the same age.50-80\% of women have at least one UTI during their lifetime, and $20-30 \%$ of women have recurrent episodes.

Bacteriology

\section{Uncomplicated UTI}

- Escherichia coli $(70-90 \%)$.

- Coagulase-negative staphylococci (S. saprophyticus) $(5-20 \%)$.

- Klebsiellaspp. $(1-2 \%)$.

- Enterococci $(1-2 \%)$.

- Proteus mirabilis $(1-2 \%)$.

- Citrobacterspp., Enterobacter spp., Pseudomonas aeruginosa, Staphylococcus aureus, group B streptococci (all <1\% each).

\section{Complicated UTI}

- E. coli remains the most common, but Proteus, Klebsiella, Citrobacterspp., enterococci, Pseudomonas aeruginosa, Staphylococcus aureus, and group B streptococci account for a higher proportion.

The commonest causative organisms in uncomplicated bacterial UTI are Gram-negative gut organisms, particularly Escherichia coli. 
This reflects the fact that most infections reach the urinary tract via the urethra from the perineum. Complicated UTIs are caused by a broader spectrum of bacteria, including Gram- positive in addition to Gram- negative organisms and those with multiple resistance to antibiotics.

\section{Pathogenesis}

In the majority of UTIs, bacteria establish infection by ascending from the urethra to the bladder. Continuing ascent up the ureter to the kidney is the pathway for most renal parenchymal infections.

\section{Contamination}

Bacteria contaminated the lower urinary tract because of the risk factors for e.g. E. coli - they initially colonize the urethra and bladder. This trigger an inflammatory response in the urinary tract. Neutrophils are then recruited in this area. In bladder we have bacteria and neutrophils. Bacteria multiply and they are able to evade the immune system. $E$. coli for example can bind to cells in lower urinary tract and hide from immune cells. Bacteria can form biofilms. A biofilm is any group of organisms in which they stick to each other and often these microorganisms adhere to surface and it allow them to survive. If this UTI progresses and left untreated or if the patient is immune-compromised has risk factors the bacteria can ascend towards the kidneys and colonies the kidneys causing an upper urinary tract infection. From here if left untreated the bacteria can spread into circulation via the renal veins causing worse caseSeptic shock.

\section{Catheterisation}

A big risk factor for developing UTI especially in female is urinary catheter. Same thing happens the catheter can introduce infection straight into bladder if not done hygienic. Bacteria colonies the bladder initiating an immune response. Neutrophils enter further promoting inflammation. Fibrinogen accumulates on the catheter providing an ideal environment for the attachment of uropathogens that express fibrinogen binding proteins. Bacteria can multiply and they can form biofilm, promote epithelial damage and concede infection of the kidneys as mentioned above.

\section{Pregnancy}

In pregnancy UTI are common. Catheterisation will almost always result in an UTI. Reason being is not only urethra shorter in women but during pregnancy progesterone relaxes smooth muscles causing stasis of urine which allows for colonisation especially up to the kidneys. During pregnancy it is important to perform urinalysis because not only are urinary tract infections are more common but also asymptomatic untreated UTI during pregnancy can have consequences for growing infant in utero.

Risk factors include recent use of a diaphragm with spermicide, frequent sexual intercourse, catheterisation, immunosuppression, a history of UTI, diabetes mellitus, urinary tract obstruction and incontinence.

\section{Signs and symptoms}

When a UTI is suspected, the most important issue is to classify it as asymptomatic bacteriuria; as uncomplicated cystitis, pyelonephritis, or prostatitis; or as complicated UTI.
- Asymptomatic bacteriuria is diagnosed when a screening urine culture performed for a reason unrelated to the genitourinary tract is incidentally found to contain bacteria, but the pt. has no local or systemic symptoms referable to the urinary tract.

- Cystitis presents with dysuria, urinary frequency, and urgency; nocturia, hesitancy, suprapubic discomfort, and gross hematuria are often noted as well. Unilateral back or flank pain and fever are signs that the upper urinary tract is involved.

- Pyelonephritis presents with fever, lower-back or costovertebral-angle pain, nausea, and vomiting. Bacteremia develops in $20-30 \%$ of cases.

- Prostatitis can be either infectious or non-infectious; non-infectious cases are far more common. Acute bacterial prostatitis presents with dysuria, urinary frequency, fever, chills, symptoms of bladder outlet obstruction, and pain in the prostatic, pelvic, or perineal area.

- Complicated UTI presents as symptomatic disease in a man or woman with an anatomic predisposition to infection, with a foreign body in the urinary tract, or with factors predisposing to a delayed response to therapy.

- Clinical Presentation - lower urinary tract infection

- Dysuria

- Frequency

- Urgency

- Nocturia

- Haematuria

- Suprapubic discomfort

- Clinical Presentation - upper urinary tract infection

- Fever

- Nausea/Vomiting

- Diarrhoea

- General malaise

Classical triad of upper urinary tract infection - vomiting, flank pain, fever.

\section{Diagnosis}

The clinical history itself has a high predictive value in diagnosing uncomplicated cystitis; in a patient presenting with both dysuria and urinary frequency in the absence of vaginal discharge, the likelihood of UTI is $96 \%$.

\section{Investigation}

Dipstick urine: positive leucocyte esterase 9 nitrite reductase. Positive predictive value of $766 \%$ and a negative predictive value of $790 \%$. May be modest haematuria or proteinuria. In low-risk cases, no further investigation is needed if positive dipstick with characteristic symptoms.

- Clean-catch MSU for microscopy at x 40 magnification (i.e. high-powered field), pyuria (>10 4 WCC per mL urine) 9 organisms. WCC casts strongly suggest pyelonephritis.

- Culture: sample should be cultured within $2 \mathrm{~h}$ of sampling. If not possible, store at $4 *$ (for $<48 \mathrm{~h}$ ). Repeat urine culture to confirm eradication post-treatment is not recommended.

- A pregnancy test should be undertaken if appropriate.

- If suspected pyelonephritis: WCC, CRP, SCr, U\&E.

- Imaging is only required in complicated cases, e.g. fever persists $>48 \mathrm{~h}$ despite therapy, clinical 
deterioration, poorly controlled diabetes, immune compromised, systemic sepsis, recurrent episodes, stone former.

- In males, assess the prostate size and bladder emptying.

- Cystoscopy may be indicated in those at risk for bladder or prostate cancer, as well as those with evidence of impaired bladder emptying who may benefit from urethral dilatation.

\section{Homeopathic remedies}

Apis

Urine incontinence, suppressed, loaded with casts; frequent and involuntary; stinging pain and Strangury, scanty; high coloured. Last drops burn and smart.

\section{Cantharis}

Burning, cutting pain in the whole renal region. Intolerable, painful urging to urinate, bloody urine, urine scalds and is passed, drop by drop. Urine scanty. Violent tenesmus and strangury.

\section{Equisetum hyemale}

Frequent and intolerable urging to urinate, with severe pain at close of urination, large quantity of clear, wateryurine, without amel. Sharp, burning, cutting pain in urethra while urinating.

\section{Sarsaparilla}

Severe, almost unbearable pain at conclusion of urination. Urine scanty, slimy, flaky, Sandy, bloody. Child screams before and while passing urine. Painful distension and tenderness in bladder. Urine dribbles while sitting, passes freely standing.

\section{Medorrhinum}

Painful tenesmus of bladder and bowels when urinating. Pain in renal region, profuse urination relieves. Urine high coloured. Extreme pain and burning in urethra on urinating.

\section{Sepia}

Chronic cystitis, slow micturition, with bearing down sensation above pubis. Pressure on bladder, urging to urinate, urine passing only after waiting burning cutting pain when urinating. Urine deposits a reddish clay coloured sediment. Urine offensive.

\section{Staphysagria}

Urging to urinate, has to sit at urinal for hours; in young married women; after coition; after difficult labour/ operation; catheterisation. Burning in urethra when not urinating. Urging and pain after urinating in prostatic troubles of old men.

\section{Conclusion}

Homoeopathic medicine show effective results in the treatment urinary tract infection, reduces its reoccurrence frequency and further reduce any chances of complications when prescribed according to the principles of Homoeopathy. This article is an attempt to explain the urinary tract infection in brief and the most common Homoeopathic medicines for its treatment.

\section{References}

1. Dan Longo, Anthony Fauci, Dennis Kasper, Stephen Haider, Jameson J, Joseph Loscalzo. Harrison's Manual of Medicine, $18^{\text {th }}$ edition: The McGraw- Hill companies. Inc 2012.

2. David Warrel A, Timothy Cox M, John Firth D. Oxford Textbook of Medicine. $6^{\text {th }}$ edition: Oxford University press 2010.

3. Simon Steddon, Neil Ashman, Alistair cheser, John Cunningham. Oxford Handbook of Nephrology and Hypertension. 2nd edition: Oxford University press 2014.

4. Brian Walker R, Nicki Colledge R, Stuart Ralston H, Ian Penman D. Davidson's, Principles and practice of medicine, $22^{\text {nd }}$ ed.. Adi BS. Efficacy of homoeopathic medicines in chronic low back pain: a clinical study. International Journal of Alternative and Complementary Medicine 2020, 17-20. Churchill Livingstone Elsevier 2014.

5. Boericke W. Boericke's new manual of Homoeopathic Materia Medica with Repertory, third revised and augmented edition based on $9^{\text {th }}$ ed.: B. Jain large print 2010.

6. Allen HC. Keynotes and Characteristics, $8^{\text {th }}$ ed.: B Jain publishers 2009.

7. https://armandoh.org/disease/urinary-tract-infection/ 